\title{
Tailoring surface charge and wetting property for robust oil-fouling mitigation in membrane distillation
}

\author{
Zhangxin Wang ${ }^{\mathrm{a}}$, Jian Jin ${ }^{\mathrm{b}}$, Deyin Hou ${ }^{\mathrm{c}}$, Shihong Lin ${ }^{\mathrm{a}, \mathrm{d}, *}$ \\ a Department of Civil and Environmental Engineering, Vanderbilt University, Nashville, Tennessee 37235-1831, United States \\ ${ }^{\mathrm{b}}$ Suzhou Institute of Nano-Tech and Nano-Bionics, Chinese Academy of Sciences, Suzhou 215123, PR China \\ ' State Key Laboratory of Environmental Aquatic Chemistry, Research Center for Eco-Environmental Sciences, Chinese Academy of Sciences, Beijing 100085, \\ PR China \\ d Department of Chemical and Biomolecular Engineering, Vanderbilt University, Nashville, Tennessee 37235-1831, United States
}

\section{A R T I C L E I N F O}

\section{Article history:}

Received 6 April 2016

Received in revised form

4 June 2016

Accepted 7 June 2016

Available online 10 June 2016

Keywords:

Membrane distillation

Oleophobic membrane

Oil fouling

Interfacial tension

Surface charge

\begin{abstract}
A B S T R A C T
The efforts of developing anti-oil-fouling membranes have so far been focusing on tailoring membrane surface wetting properties, whereas the impacts of surface charge are often eclipsed. In this study, we investigated the impacts of surface charge and wetting property on oil fouling kinetics in membrane distillation (MD). Two composite membranes with in-air hydrophilic and underwater oleophobic surfaces, one of positive charge and the other of negative charge, were fabricated by modifying a hydrophobic polyvinylidene fluoride (PVDF) membrane with hydrophilic polyelectrolytes with different charges. The modified composite membranes were compared with the reference PVDF membrane for their contact angles, adhesion force curves, and fouling kinetics in MD processes. It was found that the negatively charged composite membrane performed the best in mitigating fouling by the negatively charged oil emulsion, followed by the positively charged composite membrane, with the pristine PVDF membrane being the most susceptible to oil fouling in MD experiments. The results from underwater oil CA measurements and oil probe force spectroscopy corroborated the results in the fouling experiments. The impact of surface charges on oil-membrane interaction and associated mechanism were also discussed.
\end{abstract}

(c) 2016 Elsevier B.V. All rights reserved.

\section{Introduction}

Membrane distillation (MD) is an emerging membrane-based thermal desalination process capable of utilizing low-grade heat. In an MD process, a microporous hydrophobic membrane is employed as the barrier for liquid water transport and the medium for water vapor transport. The temperature difference between hot salty water (the feed solution) and cold deionized water (the distillate) results in a partial vapor pressure difference which drives the vapor to transport from the feed stream to the distillate stream, thereby desalinating the feed water [1-3]. Research interests in MD have recently grown significantly due to the increasing demand for desalination and the potential of MD to become a competitive technology in certain challenging desalination applications. For example, a distinct advantage of MD is the ability to harness low-grade thermal energy such as waste heat, geothermal energy, and solar thermal energy for desalination [4-6]. Such an advantage, together with the small system footprint and

\footnotetext{
* Corresponding author.

E-mail address: shihong.lin@vanderbilt.edu (S. Lin).
}

low capital cost, renders MD an appealing process for sustainable and off-grid desalination[7-11]. On the other hand, MD has also been identified as one of the only few promising technological candidates for desalinating shale gas produced water which, in certain cases, is too saline for reverse osmosis to treat [12-14].

Most existing MD membranes are only applicable to desalinate relatively 'clean' water (e.g. seawater) with a very low concentration of hydrophobic contaminants. This is because the commercially available hydrophobic membranes are inherently susceptible to fouling by hydrophobic contaminants due to the strong and long-range hydrophobic-hydrophobic interaction [15-17]. The direct consequence of MD membrane fouling is flux decline attributed to the blocking of membrane pores by the accumulated foulants. This problem of membrane fouling by hydrophobic contaminants is particularly conspicuous when MD is employed to desalinate saline industrial wastewaters enriched in hydrophobes. One prominent example is shale gas produced water, which is not only hyper-saline but in many cases abundant with hydrocarbons. Previous MD studies using a hydrophobic polyvinylidene fluoride (PVDF) membrane to treat a feed solution with oil reported rapid flux decline that led to process failure in a relatively short time $[18,19]$. Another example is the application of MD to treat 
anaerobic digestate which, though not as potent as oil emulsion as a foulant, still led to considerable flux decline in a long term operation [20].

An effective approach to mitigate oil fouling in MD is to develop membranes with an underwater oleophobic surface that resists oil adhesion when submerged in water. The underwater oleophobicity is to be distinguished from the in-air oleophobicity which stands for resistance to oil adhesion in air. Inspired by biological surfaces such as sharkskin and clamshells that are resistant to biological or organic adhesion underwater, the materials science community has developed a plethora of interfacial materials with underwater superoleophobicity for different applications such as marine biofouling control $[21,22]$ and oil-water separations [23-33]. In general, there are two requirements for constructing an underwater superoleophobic surface: rough (textured) surface morphology and hydrophilic surface chemistry. Following this principle, composite MD membranes with a hydrophobic substrate and an underwater oleophobic top surface have been developed using hydrogel-nanoparticle composites for oil fouling mitigation $[18,19]$. The presence of highly hydrophilic surface coating renders the feed-facing surface of the MD membrane in-air superhydrophilic and underwater superoleophobic, enabling MD to desalinate oily feed water with robust anti-fouling performance.

It is well known in surface science that a hydrogen-bond forming, superhydrophilic surface can resist the adhesion of hydrophobic substances underwater due to the formation of a surface hydration layer that serves as an energetic barrier for hydrophobic foulants to attach onto substrate surface [34-38]. This mechanism, namely the hydration force, has been a pivotal guiding principle for developing fouling resistant membranes and antioil-adhesion surfaces $[39,40]$. Although the effect of hydration force is indeed prominent, there is another important factor that plays a critical role in oil-membrane interaction but is nonetheless often neglected in developing underwater oelophobic surfaces $[28,41-43]$. This factor is surface charge, which plays a significant role in controlling both electrical double layer (EDL) interaction and interfacial tension $[44,45]$.

In a recent study where a PVDF MD membrane was coated with titanium dioxide $\left(\mathrm{TiO}_{2}\right)$ nanoparticles and polyethylene glycol (PEG) to acquire resistance to mineral oil fouling, it has been observed that varying the $\mathrm{pH}$ around the isoelectric point of the modified PVDF slightly affected the fouling kinetics [19]. However, such an effect was not appreciable, which may be explained by the fact that both the modified PVDF membrane and the mineral oil emulsion had similar isoelectric points and their interaction was thus always repulsive regardless of the solution $\mathrm{pH}$. On the other hand, many studies conducted to develop underwater superoleophobic meshes or membranes for oil-water separation only focused on engineering the surface wetting properties but largely overlooked the impact of the surface charge. Because both the electrostatic interaction and the hydration force may be important as suggested by the extended Derjaguin Landau Verwey Overbeek ( $x$-DVLO) theory [46], it is our objective in this study to systematically assess the impacts of these two interactions on the oilmembrane interaction and consequently on the propensity of an MD membrane to be fouled by an oil-in-water emulsion.

In this study, we compare the performance of three membranes with different surface wetting and charge properties in direct contact membrane distillation (DCMD) experiments with feed water containing crude oil. A hydrophobic and negatively charged PVDF membrane is used as the reference. The other two membranes are composite membranes prepared by modifying the hydrophobic PVDF membranes with nanocomposite coating comprising silica nanoparticles and hydrophilic polymers with different surface charges. We characterize these membranes to acquire information regarding their morphological and wetting properties and conduct DCMD experiments with these membranes to compare their fouling kinetics. We also conduct force spectroscopy using a force tensiometer to probe the underwater interactions between a crude oil droplet and different membranes, and apply these force curves to elucidate the difference of these membranes in their fouling propensity.

\section{Materials and methods}

\subsection{Materials and chemicals}

The hydrophobic microporous membranes, used as the reference in fouling experiments and as the substrates for surface modification, were made of PVDF with a nominal pore size of $0.45 \mu \mathrm{m}$ (GE Healthcare Life Sciences, Pittsburg, PA). Dopamine hydrochloride, Trizma hydrochloride buffer, poly(diallyldimethylammonium chloride) solution (PDDA, $20 \%$ in $\mathrm{H}_{2} \mathrm{O}$ ), acetone, sodium chloride $(\mathrm{NaCl})$, potassium chloride $(\mathrm{KCl})$, sodium hydroxide $(\mathrm{NaOH})$, and hypochloric acid $(\mathrm{HCl})$ were all procured from Sigma Aldrich (St. Louis, MO). Silica nanoparticles (SiNPs) with diameters ranging between 20 and $60 \mathrm{~nm}$ were purchased from SkySpring Nanomaterials (Houston, TX). The crude oil as the fouling agent was acquired from Texas Raw Crude Oil (Midland, TX).

\subsection{Fabrication of the underwater oleophobic membranes}

We prepared two different composite membranes by modifying the PVDF substrate membrane. Both membranes were underwater oleophobic due to the presence of the highly hydrophilic coating. However, due to the different hydrophilic surface modifiers used, the two membranes were of opposite charges at environmentally relevant $\mathrm{pH}$. This subsection describes the experimental details of fabricating the two composite membranes.

\subsubsection{Composite membrane coated with polydopamine (PDA) and SiNPs}

The first composite membrane was prepared by coating a PVDF membrane with a layer of negatively charged nanocomposite of PDA and SiNPs (PDA/SiNPs). We prepared such a composite membrane by first spray-coating the substrate membrane with SiNPs and then dip-coating the SiNPs coated membrane with PDA $[47,48]$. The SiNPs, which are inexpensive and chemically inert, were used to increase the surface roughness and create hierarchical morphology that are essential for in-air superhydrophilicity and underwater superoleophobicity. For spraycoating, $0.5 \mathrm{~g}$ SiNPs were dispersed in $100 \mathrm{~mL}$ acetone under sonication for $5 \mathrm{~min}$ to obtain a $5 \mathrm{~g} / \mathrm{L}$ SiNPs suspension. $20 \mathrm{~mL}$ of SiNPs suspension were spray-coated onto a PVDF membrane substrate with an area of $20 \mathrm{~cm} \times 10 \mathrm{~cm}$ using a spray gun with $0.2 \mathrm{MPa}$ air.

After briefly dried in air, the SiNPs coated membrane was submerged in a dopamine solution prepared by dissolving $0.1 \mathrm{~g}$ dopamine powder in $100 \mathrm{~mL}$ of a $15 \mathrm{mM}$ Trizma hydrochloride buffer solution with the $\mathrm{pH}$ adjusted to 8.5. A layer of PDA formed as a result of self-polymerization of dopamine, covering the SiNPs and the top surface of the PVDF membrane. During the dip-coating process, the PVDF membrane was taped around on a glass substrate to ensure that only the SiNPs coated surface of the membrane was exposed to the dopamine suspension. Because the hydrophilic PDA solution could not wet the hydrophobic pores of the PVDF membrane, the hydrophobicity of the inner pore structure of the PVDF substrate was not compromised, i.e., only the wetting property of the membrane surface, not that of the entire membrane, was altered by this membrane modification approach. After 
24-hour self-polymerization of dopamine, the PDA/SiNPs coated PVDF (PDA/SiNPs-PVDF) membrane was washed with DI water and dried at room temperature.

\subsubsection{Composite membrane coated with PDDA and SiNPs}

The second composite membrane with PDDA and SiNPs nanocomposite (PDDA/SiNPs) was fabricated via layer-by-layer spray-coating [49]. All the spray-coating steps were conducted using a spray gun with $0.2 \mathrm{MPa}$ pressurized air. First, a PVDF membrane substrate with an area of $20 \mathrm{~cm} \times 10 \mathrm{~cm}$ was spraycoated a layer of PDDA with $5 \mathrm{~mL}$ aqueous PDDA solution $(52 \mathrm{~g} / \mathrm{L})$ and then dried at room temperature. Second, $10 \mathrm{~mL}$ SiNPs suspension ( $5 \mathrm{~g} / \mathrm{L}$ in acetone) was sprayed onto the PDDA coated membrane to form a SiNPs layer. The function of first PDDA layer was to impart positive charge to the PVDF substrate so that the SiNPs can attach onto the PDDA coated membrane via electrostatic interaction. Lastly, the air-dried SiNPs decorated membrane was spray-coated with another layer of PDDA solution ( $1 \mathrm{~mL}, 52 \mathrm{~g} / \mathrm{L})$. The resulting membrane was heated at $100{ }^{\circ} \mathrm{C}$ for $1 \mathrm{~h}$. The coating applied using this approach has been tested to be mechanically robust [49].

\subsection{Characterizations of morphology and wetting property}

The surface morphology of the pristine PVDF membrane and the two fabricated composite membranes was observed using scanning electron microscopy, or SEM (Merlin, Zeiss, Thornwood, NY) and atomic force spectroscopy, or AFM (Dimension Icon, Bruker, Billerica, MA). The pore size distribution and the liquid entry pressure, LEP, of each membrane were determined with a capillary flow porometer (Poroflux 1000, IB-GT GmbH, Germany) [50-53]. In addition, the chemical composition of each membrane surface was confirmed via polarization modulation-infrared reflection-adsorption spectroscopy, or PM-IRRAS (PMA 50, Bruker, Billerica, MA).

To compare the wetting properties of the three membranes, the static contact angle (CA) was measured using an optical tensiometer (TL100, Attension, Finland). For each membrane, both the in-air sessile drop CA with water and underwater CA with crude oil were measured. The measurements of underwater $C A$ with crude oil were similar to measuring captive bubble CA but with the bubble replaced by a floating crude oil droplet. Each contact angle was measured at five different locations on the membrane sample.

\subsection{Preparation and characterization of oily feed solution}

The saline crude oil emulsion (1000 ppm wt\%) was prepared by mixing $2 \mathrm{~g}$ of crude oil with $2 \mathrm{~L}$ of $\mathrm{NaCl}$ aqueous solution $(0.6 \mathrm{M})$ at $16,000 \mathrm{rpm}$ for $15 \mathrm{~min}$ using a homogenizer (Fisher Scientific, Waltham, MA). The morphology of the oil droplets in the prepared solution was observed using an optical microscope (Nikon LV100, Japan). The size distribution and zeta potential of the oil droplets in the emulsion were determined by dynamic light scattering (DLS) and electrophoresis, respectively, both with a Zetasizer (Malvern Nano ZS, UK). The $\mathrm{pH}$ of the prepared feed solution was also measured.

\subsection{Membrane zeta potential measurement}

We measured the zeta potential of the pristine PVDF membranes and the composite membranes using a streaming potential analyzer (SurPASS, Anton Paar, Ashland, VA). In these measurements, the membrane coupons were taped onto two parallel and opposing planar surfaces across a slit with adjustable width. The electrolyte solution flowed, in alternating directions, along the slit composed of the two parallel surfaces covered by the membrane samples. This flow of electrolyte solution generated streaming potentials from which the zeta potential of the membrane surface was calculated using the Helmholtz-Smoluchowski equation $[54,55]$. The electrolyte solution was $1 \mathrm{mM} \mathrm{KCl}$ and the $\mathrm{pH}$ was automatically adjusted by the addition of $\mathrm{KOH}$ and $\mathrm{HCl}$.

\subsection{Membrane fouling tests}

DCMD experiments were conducted with the saline crude oil emulsion to compare the fouling behaviors of the pristine PVDF membrane and the fabricated composite membranes. In these experiments, membrane coupons with an area of $2.5 \mathrm{~cm} \times 8 \mathrm{~cm}$ were used in a cross-flow test cell using a co-current flow configuration. In the experiments with composite membranes, the modified, underwater oleophobic surfaces of the membranes were in contact with the feed solution, whereas the unmodified sides faced the distillate. The influent temperatures of the oily feed solution and deionized distillate were $60{ }^{\circ} \mathrm{C}$ and $20^{\circ} \mathrm{C}$, respectively.

To facilitate the detection of membrane wetting, the flowrates of feed and distillate streams were controlled to be $0.45 \mathrm{~L} / \mathrm{min}$ and $0.2 \mathrm{~L} / \mathrm{min}(9.6 \mathrm{~cm} / \mathrm{s}$ and $4.3 \mathrm{~cm} / \mathrm{s}$ in our test cell), respectively $[13,18]$. This operation conditions resulted in a slightly higher hydraulic pressure in the feed stream than in the distillate stream in this specific test cell, so that the liquid flow through any potentially wetted pores would always be driven from the feed stream to the distillate stream and thereby increase the salinity of the distillate stream. The mass and conductivity of the solution in the distillate reservoir were constantly measured and recorded. The water vapor flux and salt rejection were calculated from the temporal change of mass and conductivity of the distillate.

\subsection{Underwater oil-adhesion force measurements}

To mechanistically understand the fouling behaviors of the pristine PVDF membrane and the fabricated composite membranes, force spectroscopy was conducted with a force tensiometer (Sigma701, Attention, Finland) to probe the underwater interaction between a crude oil droplet and the membrane surfaces [25,56-58]. In these force spectroscopic experiments, the membrane samples were fixed at the bottom of a transparent test cell filled with electrolyte solution. A small droplet $(\sim 6 \mu \mathrm{L})$ of crude oil was carefully hung on to a submerged force probe (T114, Attention, Finland) using a syringe. For collecting the force curves, the force probe with the attached oil droplet approached the membrane sample surface at a constant speed of $0.3 \mathrm{~mm} / \mathrm{min}$ until the oil droplet contacted with the membrane surface. Once physical contact between the oil droplet and the membrane sample occurred, the force probe retracted from the membrane surface at the same speed as approaching. Compressing the oil droplet against the sample surface was deliberately avoided, because the force probe was an open ring that can be readily forced into the crude oil phase and generate data that is not directly relevant to the oil-membrane interaction. The results from the force spectroscopy were force curves that quantify the force, measured by the micro-electro-mechanical sensor, as a function of the force probe position.

\section{Experimental results}

\subsection{Membrane surface properties}

\subsubsection{Membrane surface morphology}

The SEM and AFM images of the pristine PVDF membrane (Fig. 1(A), (D) and (G)), the PDA/SiNPs-PVDF membrane (Fig. 1(B), 


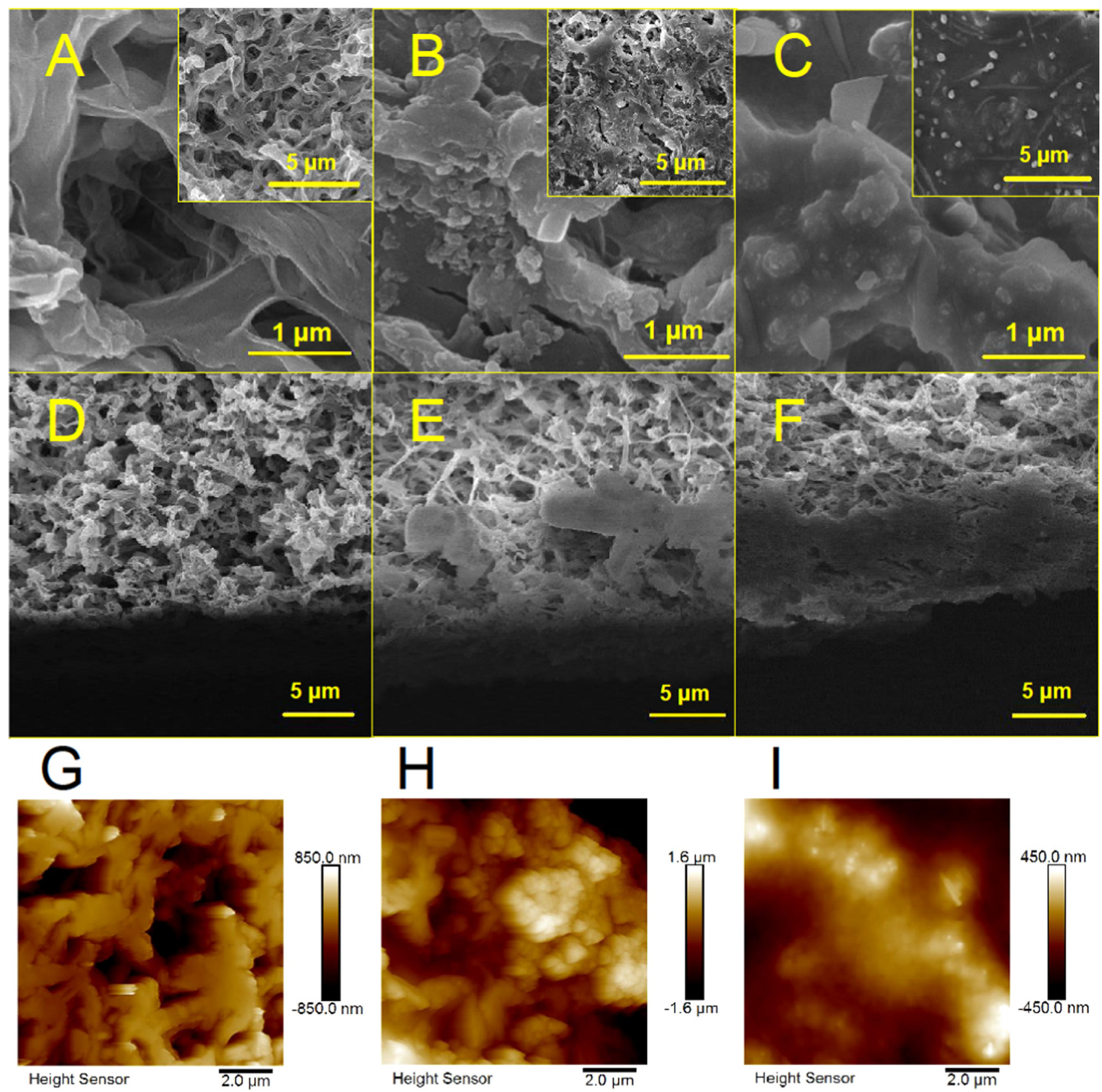

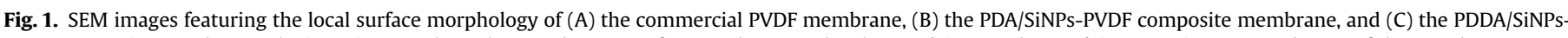

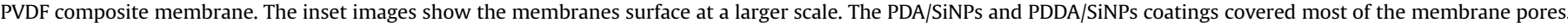

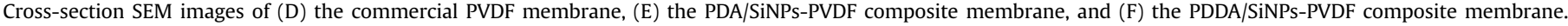
Surface AFM images of $(G)$ the commercial PVDF membrane, $(H)$ the PDA/SiNPs-PVDF composite membrane, and (I) the PDDA/SiNPs-PVDF composite membrane.

(E) and (H)), and the PDDA/SiNPs-PVDF (Fig. 1(C), (F) and (I)), show that surface modifications drastically affected the morphology of the PVDF membranes. Specifically, both the top view (Fig. 1(B) and (C)) and the cross-section view (Fig. 1(E) and (F)) of the SEM images suggest that the nanocomposite coatings thoroughly cover the surface of the PVDF substrate and block most of the membrane pores on the surface. However, the modifications did not significantly affect the pore size distribution (Fig. A1) of the membrane because the thickness of coating layer is very small compared to the thickness of the PVDF membrane which was $176.4 \pm 7.3 \mu \mathrm{m}$.

The surface modifications did not lead to any appreciable change in LEP either. Compared to the PVDF membrane with an LEP of 2.93 bar, the LEP of the PDDA/SiNPs and PDA/SiNPs modified PVDF membranes were 2.98 and 3.01 bar, respectively.
Although the surface modification significantly reduced the size of the membrane surface pores, the strong hydrophilicity of the coating layer facilitated the permeation of water with little extra resistance. Consequently, the LEP should still be primarily determined by the wetting property and pore size of the hydrophobic substrate which in all three cases were the same PVDF membrane. For the similar reason, the coated membranes still maintained significant water vapor permeability, which will be further discussed in Section 3.3. The hydrophilic nanocomposite PDA/SiNPs and PDDA/SiNPs coatings exhibited hierarchical roughness which, according to Wenzel theory, enhances the hydrophilicity of the surface $[59,60]$. The results from PM-IRRAS spectra confirmed the chemical compositions of the surface coatings on the modified composite membranes (Fig. A2). 


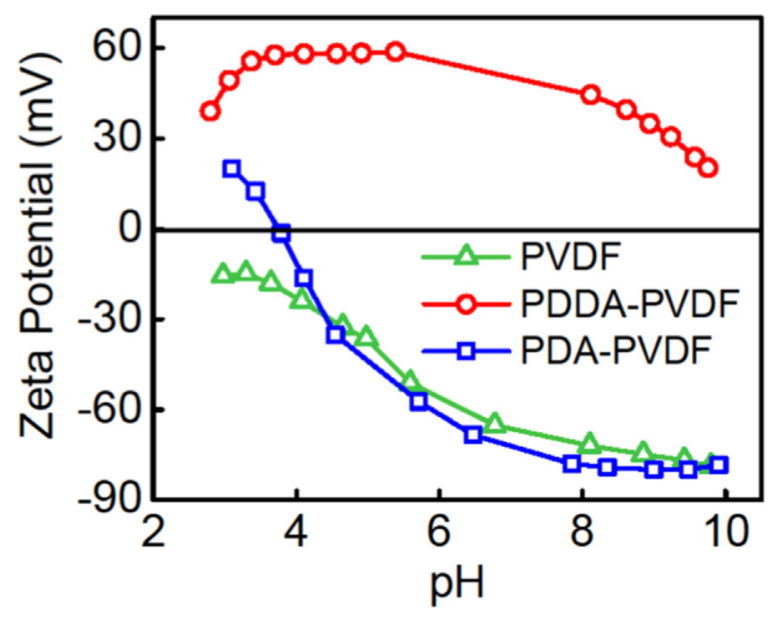

Fig. 2. Zeta potentials of the unmodified PVDF membrane (green triangles) and the PDDA/SiNPs-PVDF composite membrane (red circles) and PDA/SiNPs composite membrane (blue squares) as a function of $\mathrm{pH}$. The background electrolyte concentration was $1 \mathrm{mM} \mathrm{KCl}$. (For interpretation of the references to color in this figure legend, the reader is referred to the web version of this article.)

\subsubsection{Membrane surface potential}

Surface potential is an important membrane property affecting the electrostatic interaction between membranes and oil droplets. In this study, we measured the zeta potentials of each membrane at different $\mathrm{pH}$ (Fig. 2). The zeta potential varied with $\mathrm{pH}$ due to protonation or deprotonation of surface functional groups [61,62]. Throughout the entire $\mathrm{pH}$ range tested, the pristine PVDF membrane was negatively charged (green triangles in Fig. 2) and the PDDA/SiNPs-PVDF composite membrane was positively charged (red circles in Fig. 2). The point of zero charge (PZC) for the PDA/ SiNPs-PVDF membrane was slightly below 4, significantly lower than the $\mathrm{pH}$ of the saline crude oil emulsion, which was between $5-6$.

\subsubsection{Membrane surface wetting property}

The wetting properties of the composite membranes were drastically different from that of the pristine PVDF membrane. The in-air water CA (blue bars in Fig. 3) of the pristine PVDF, the PDA/ SiNPs-PVDF composite membrane, and the PDDA/SiNPs-PVDF

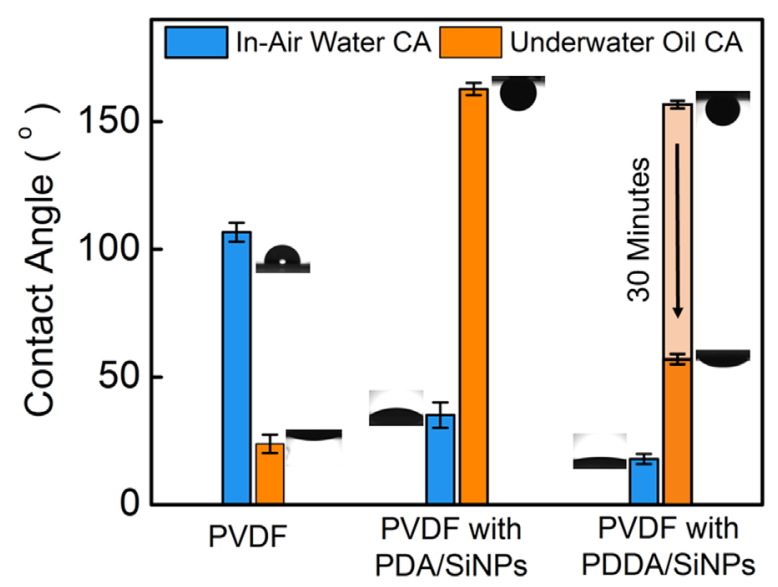

Fig. 3. Comparison of in-air water CA (blue bars) and underwater oil CA (orange bars) between the pristine PVDF membrane and the fabricated composite membranes. The images for the sessile water drop and captive oil drop are juxtaposed with the corresponding CA. The underwater oil CA for the PDDA/SiNPs-PVDF composite membrane decreased along time (shown in the bar chart) and eventually became zero as the crude oil wicked the membrane. (For interpretation of the references to color in this figure legend, the reader is referred to the web version of this article.) composite membrane were $106.7 \pm 3.7^{\circ}, 35.1 \pm 5^{\circ}$ and $17.9 \pm 2^{\circ}$, respectively. Both nanocomposite coatings imparted strong hydrophilicity to the PVDF membrane that was originally hydrophobic, which can be explained by the strong attractive interaction between water and the hydrophilic polymer on the modified membrane surfaces.

To obtain the membrane wettability information directly relevant to oil fouling in $\mathrm{MD}$, we also measured the underwater $\mathrm{CA}$ with crude oil for the three membranes (orange bars in Fig. 2). The unmodified PVDF membrane was instantly wetted by the crude oil with a negligibly small oil CA, indicating that the membrane was underwater superoleophilic. In comparison, the PDA/SiNPs-PVDF composite membrane exhibited underwater superoleophobicity with a very high oil CA $\left(162.8 \pm 2.4^{\circ}\right)$. Such underwater superoleophobicity is attributable to the strong interaction between the water and the hydrophilic surface coating (i.e., the hydration force). As a highly hydrophilic polymer, PDA has a variety of amide and alcohol functional groups that can form hydrogen bonds with water and create an interfacial hydration layer on the membrane surface $[36-38,63,64]$. Wetting the hydrogel coated membrane by the crude oil requires surface dehydration, which is energetically highly unfavorable. With a similar principle, underwater superoleophobicity was also expected for the PDDA/SiNPs-PVDF composite membrane, because PDDA is also a highly hydrophilic polymer that forms abundant hydrogen bonds with water. Indeed, the initial underwater oil CA for PDDA/SiNPs-PVDF membrane was also very high $\left(157.7 \pm 1.5^{\circ}\right)$, indicating the composite membrane was superoleophobic upon immediate contact with water. However, the oil CA decreased over time to $57.1 \pm 2^{\circ}$ in about $30 \mathrm{~min}$, which implies that the PDDA/SiNPs-PVDF composite membrane became oleophilic if sufficient time was given for the oil-membrane interaction to reach equilibrium. In comparison, the underwater oil CA for the PDA/SiNPs-PVDF membrane was very stable with no observable change in $1 \mathrm{~h}$.

Such a difference between the two composite membranes in their dynamic wetting behavior is most likely attributable to the difference in their surface charges and hence the electrostatic interaction with the crude oil. In fact, the x-DLVO theory accounts for the electrical double layer, van der Waals, and the Lewis acidbased $(A B)$ interactions, two of which depend on surface charge $[44,46]$. The $\mathrm{x}$-DLVO theory can in part explain the observed difference in the wetting behaviors between the two composite membranes, as the electrostatic interaction between the PDA/ SiNPs-PVDF membrane and the oil droplet was repulsive and that for between the PDDA/SiNPs-PVDF membrane and the oil droplet was attractive. More detailed discussion will be given in Sections 4.1 and 4.2 regarding the applicability of the $x$-DLVO theory in explaining the oil-membrane interaction.

\subsection{Properties of the saline crude oil emulsion}

The crude oil emulsion (1000 ppm wt\%), prepared as oil feed solution, was highly turbid (Fig. 4(A) inset). The emulsion was stable without observable phase separation for at least $24 \mathrm{~h}$. Such kinetic stability of the emulsion can be attributed to the presence of naturally occurring emulsifier such as asphaltenes, naphthenic acid, carboxylic acid, and other oil-soluble acids and bases in crude oil $[65,66]$.

Observation using optical microscopy suggested that the diameters of the oil droplets in the emulsion were in the range of few microns (Fig. 4(A)), which was confirmed by DLS measurements that yielded a distribution of hydrodynamic diameter of $4.9 \pm 0.8 \mu \mathrm{m}$ (Fig. 4(B)). Since the nominal pore size of the PVDF membrane substrate was only $0.45 \mu \mathrm{m}$, the oil droplets are expected to effectively block the membrane pores once they attach onto the membrane surfaces. The zeta potential of the oil droplets 

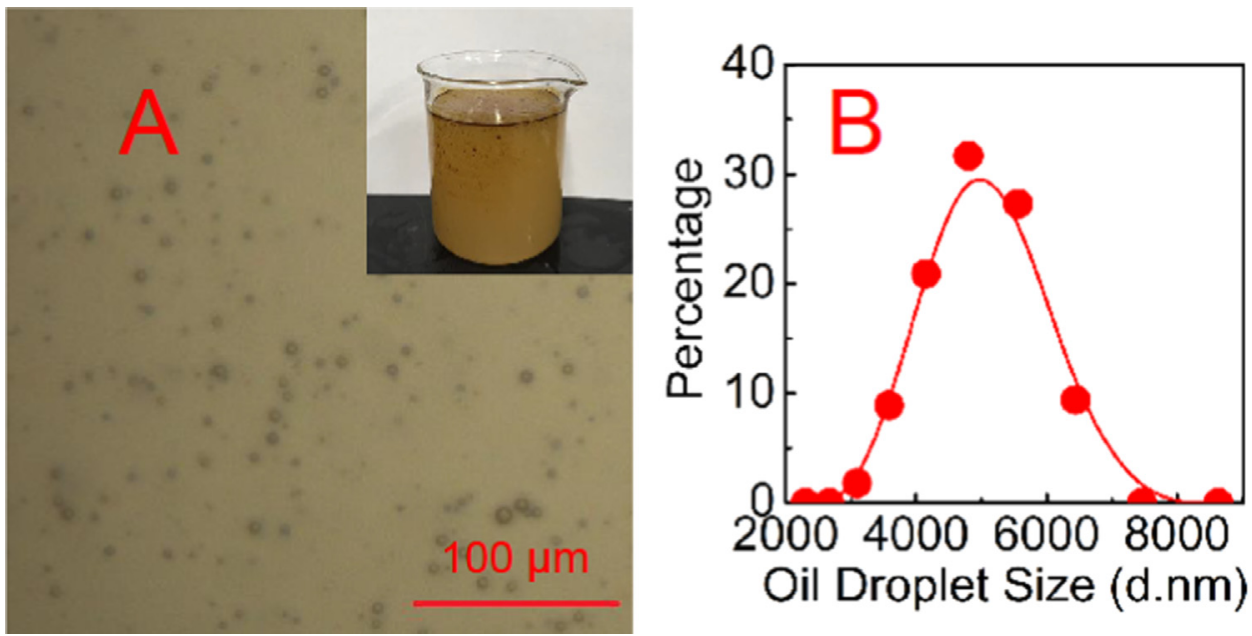

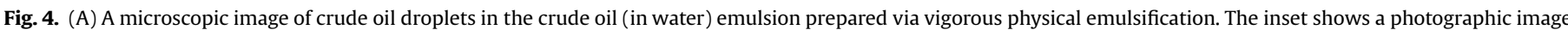

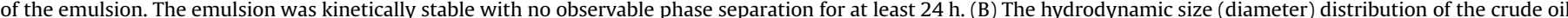
droplets measured using dynamic light scattering.

in the emulsion was $-16.4 \pm 0.8 \mathrm{mV}$ derived from the measured particle electrophoretic mobility. The $\mathrm{pH}$ of the prepared feed solution was measured to be between 5 and 6 , probably due to the various amphiphilic acids present at the water-oil interface.

\subsection{Membrane performance in MD experiments}

DCMD experiments with the saline crude oil emulsion using the pristine PVDF membrane and the two composite membranes showed drastically different fouling behaviors (Fig. 5). In each experiment, the conductivity of the distillate was observed to be decreasing with more feed water recovered, yielding a salt rejection rate of over $99.9 \%$ for every membrane tested. The fouling of the pristine PVDF membrane was very fast as indicated by drastic decline of water vapor flux in a short period of time (green triangles in Fig. 5). In fact, the water flux dropped to almost zero in

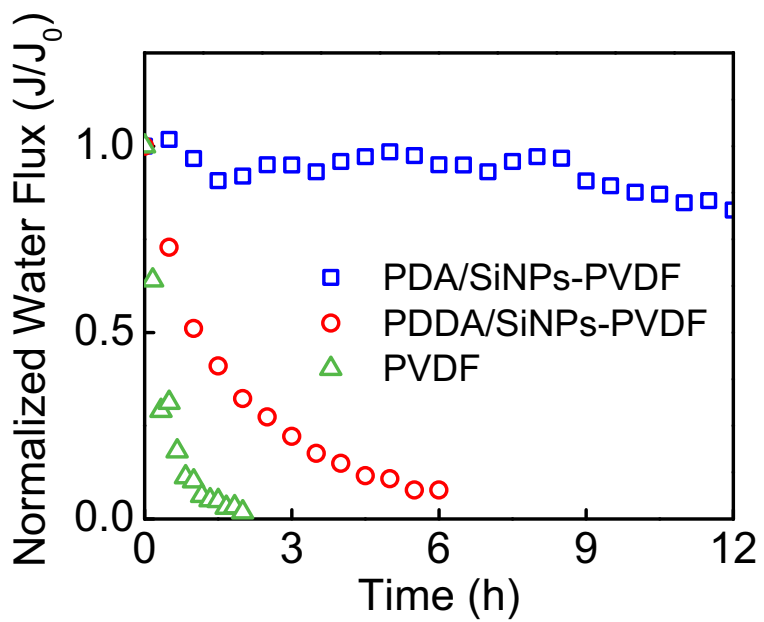

Fig. 5. Normalized water (vapor) flux for PDA/SiNPs-PVDF composite membranes (blue squres), PDDA/SiNPs-PVDF composite membrane (red circles), and pristine PVDF membrane (green triangles) with a saline feed solution containing $1000 \mathrm{ppm}$ (\% wt) crude oil in the DCMD experiments. The normalized water fluxes were the time-dependent water fluxes normalized by the initial water fluxes of the respective membranes. The cross flow velocities in the feed stream and distillate stream were $9.6 \mathrm{~cm} / \mathrm{s}$ and $4.3 \mathrm{~cm} / \mathrm{s}$, respectively. The feed and distillate temperatures were $60{ }^{\circ} \mathrm{C}$ and $20^{\circ} \mathrm{C}$, respectively. The initial water fluxes for PVDF membrane and composite membranes with PDA/SiNPs and PDDA/SiNPs were $30.96 \mathrm{~L} \mathrm{~m}^{-2} \mathrm{~h}^{-1}$, and $24.29 \mathrm{~L} \mathrm{~m}^{-2} \mathrm{~h}^{-1}$ and $23.6 \mathrm{~L} \mathrm{~m}^{-2} \mathrm{~h}^{-1}$, respectively. (For interpretation of the references to color in this figure legend, the reader is referred to the web version of this article.) only about two hours. Since the PVDF membrane was in-air hydrophobic and underwater oleophilic as suggested by the CA measurements, the very strong long-range hydrophobic-hydrophobic interaction between the crude oil droplets and the membrane surface promoted the retention of oil droplets on the membrane surface and resulted in blockage of membrane pores that served as the pathways for water vapor flux.

In comparison, the DCMD performance of the PDA/SiNPs-PVDF composite membrane was appreciably more stable. With the same crude oil emulsion that severely fouled the pristine PVDF membrane, the PDA/SiNPs-PVDF composite membrane was able to maintain a nearly constant water vapor flux along the $12 \mathrm{~h}$ MD operation (blue squares in Fig. 5). The more robust MD performance of the PDA/SiNPs-PVDF composite membrane can be explained by the underwater superoleophobicity of the composite membrane as suggested by its underwater oil CA. The strong hydration force due to the presence of the highly hydrophilic PDA polymer, and the electrostatic repulsion between the nanocomposite and the oil droplets, both contributed to mitigating the adhesion of the oil droplets to the membrane surface.

The PDDA/SiNPs nanocomposite coating slowed down the membrane fouling as reflected by the appreciably slower flux decline of the water vapor flux (red circles in Fig. 5) as compared to that with the pristine PVDF membrane. However, the fouling control was significantly less effective with the PDDA/SiNPs nanocomposite than with the PDA/SiNPs nanocomposite. The difference in anti-fouling performance between these two nanocomposite membranes is in good accordance with the results observed in underwater oil CA measurements. The initial underwater superoleophicity of the PDDA/SiNPs-PVDF membrane, which was a result of the repulsive hydration force, contributed to the slower flux decline as compared to the pristine PVDF membrane. However, the PDDA/SiNPs-PVDF membrane gradually lost its oleophobicity possibly due to electrostatic attraction, which eventually led to significant membrane fouling within a relatively short time. In comparison, the PDA/SiNPs-PVDF was able to sustain a much more robust MD performance due to the presence of both hydration force and electrostatic repulsion.

The initial fluxes of the PDDA/SiNPs-PVDF composite membrane (23.6 LMH) and PDA/SiNPs-PVDF composite membrane (24.3 LMH) were lower than that of the pristine PVDF membrane (31.0 LMH). The flux reduction was obviously consequent of the surface modification that blocked a significant portion of the membrane. On the other hand, the percentages of flux reduction 
were clearly not in proportion to the fraction of pore blockage shown in the SEM images. Because the nanocomposite layers were porous hydrophilic networks, the liquid feed water should be able to permeate through layer with limited resistance. We therefore speculate that the reduction of vapor flux was mostly attributable to the enhanced temperature polarization in the coating layer. The hydrated nanocomposite coating is a hydraulically stagnant layer insusceptible to the cross-flow induced mixing in the feed channel $[2,67,68]$. Similar to the internal concentration polarization in forward osmosis which has a detrimental impact on mass transfer [69], the temperature polarization across the hydrated nanocomposite layer was also "internal" in the sense that it occurred within the membrane structure. This additional temperature polarization served to reduce the driving force for vapor transfer across the MD membranes.

\subsection{Force spectroscopy with oil droplet probe}

To acquire more fundamental understanding about the interaction between the oil droplet and the membrane surface and to explain the different fouling behaviors with different membranes, we conducted force spectroscopy with an oil droplet as the force probe and water as the medium. The position dependent interaction forces between an oil droplet and different membrane surfaces are presented in Fig. 6 for comparison. A complete interaction curve includes two stages, the advancing and receding stages, and five distinct events, including "approach", "contact", "retract", "split" and "detach". The details for general interpretation of the force curves were given in our previous publication [18].

Before the oil droplet force probe contacted the surface, the "adhesion force" was set to zero by default, as there was no

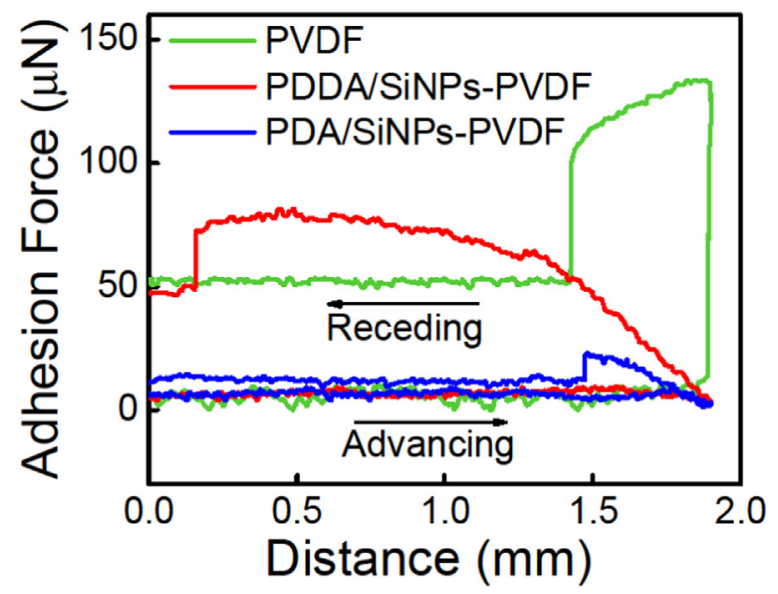

Fig. 6. Force curves for the interactions of a crude oil droplet with the pristine PVDF membrane (green curve), the PDA/SiNPs-PVDF membrane (blue curve) and the PDDA/SiNPs-PVDF membrane (red curve). The advancing stage was represented by the flat baselines with close-to-zero adhesion force measured when the oil droplet probe approached the membrane surface (the bottom parts on the force curves, proceeding from left to right). There was no difference between the three tested membranes in the force curves during the advancing stage since no interaction with the membrane had occurred. The remaining parts of the force curves were obtained from the receding stage in which the oil droplet probe was pulled away from the surface (proceeding from right to left on the figure). This part of the force curve was distinctive for each membrane as it was strongly dependent on the oil-membrane interaction. A precipitous drop in adhesion force was present in every single force curve, representing the split of the oil droplet probe into two parts with one remaining on the probe and the other staying on the membrane surface. A constant "adhesion force" was measured, without exception, in the end of the each force curve because the oil droplet probe no long interacted with the membrane after the split. The non-zero and constant "adhesion forces" at the end of all force curves were nothing but an artifacts (i.e. they do not represent actual adhesion), resulting from the change of oil droplet volume and thus the forces measured by the tensiometer. (For interpretation of the references to color in this figure legend, the reader is referred to the web version of this article.) physical interaction between the oil droplet and the membrane. Upon contact between the oil droplet force probe and the pristine PVDF membrane, a strong and sudden attraction was detected. This strong attraction can be attributed to the hydrophobic interaction between the oil droplet and the PVDF membrane $[15,16,70]$. As the force probe retracted, it experienced an attractive force until the split of the oil droplet. The oil droplet split was reflected on the force curve as a precipitous drop in the "adhesion force".

A finite "adhesion force" was measured after the split of the oil droplet, which was an artifact resulting from a smaller oil droplet remaining on the force probe. The new "baseline" was non-zero because the difference between buoyancy and gravity for the remaining oil droplet was smaller than that of the original oil droplet [18]. However, there was no physical oil-membrane interaction or "adhesion" corresponding to the finite "adhesion force", because the oil droplet had already detached from the surface. Although such an "adhesion force" measured by the tensiometer was an artifact in essence, its magnitude positively correlates with the size of the oil droplet remaining on the force probe, which still yields a useful piece of information regarding the affinity between oil droplet and the membrane surface.

For the PDA/SiNPs-PVDF composite membrane, no strong and sudden attraction between the oil droplet and the membrane was observed. On the contrary, a slight repulsive interaction was detected as suggested by a negative "adhesion force" measured upon the contact between the oil droplet and the composite membrane. We did not attempt to quantify such a repulsive force by further pressing the oil probe against the membrane, as the ring-shaped force probe could be forced into the compressed oil droplet and generates irrelevant interaction information. Therefore, the force probe was retracted almost instantly after the oil droplet contacted the membrane.

Nonetheless, the repulsion between the oil droplet and the PDA/SiNPs-PVDF composite membrane upon their first contact was conspicuous from the recorded force curve. The retraction of the force probe resulted in attractive interaction between the oil droplet and the membrane as evidenced by the increasing adhesion force during the receding stage, before the oil droplet split. However, such an adhesion force was very small for the PDA/ SiNPs-PVDF composite membrane, as revealed by the force curve (blue curve in Fig. 6). Furthermore, the remaining part of the force curve after oil droplet split only shifted slightly from the original baseline, indicating that only a small proportion of the oil droplet remained on the membrane surface.

The shape of the force curve for the interaction of an oil droplet with the PDDA/SiNPs-PVDF composite membrane resembled that for the interaction with the PDA/SiNPs-PVDF membrane. For instance, repulsion was also observed upon the initial contact between the oil droplet and the composite membrane. However, the attractive interaction between the oil droplet and the PDDA/SiNPsPVDF was significantly stronger than that between the oil droplet and the PDA/SiNPs-PVDF membrane during the force probe retraction. Since the primary difference between the two composite membranes was the surface zeta potential, it is likely that electrostatic interaction played an important role that differentiated two composite membranes in their interactions with the oil droplet probe.

\section{Discussion}

\subsection{Implications of electrostatic interaction}

The results from the force spectroscopy can be employed to explain the different fouling behaviors observed with different MD membranes. The pristine PVDF membrane was most susceptible to 
fouling as evidenced by the fastest flux decline among three membranes. This can be explained by the instant and very strong attraction between the oil droplets and the PVDF membrane via the strong hydrophobic-hydrophobic interaction.

On the other hand, the other two underwater oleophobic membranes both mitigated membrane fouling due to the presence of the repulsive hydration force. However, the extents to which membrane fouling was alleviated by the two composite membranes were very different due to the different surface charges and consequently different electrostatic interactions. The attractive electrostatic interaction between the positively charged PDDA/ SiNPs-PVDF membrane and the negatively charged crude oil particles offset the repulsive hydration force, leading to a membrane fouling rate between that with the hydrophobic and underwater oleophilic PVDF membrane and that with the underwater oleophobic and negatively charged PDA/SiNPs-PVDF membrane.

The response for the wettability-based interaction seemed to be much faster than that for the electrostatic interaction. This phenomenon was observed in both the underwater oil CA measurements and the oil droplet probe force spectroscopy. In the underwater oil CA measurement using the PDDA/SiNPs-PVDF membrane, the initial underwater oil CA was very high due to the in-air hydrophilic surface coating of the PDDA/SiNPs. The gradual transition of the membrane surface from being underwater superoleophobic to underwater oleophilic can be attributed to electrostatic interaction which did not take effect immediately after the initial oil-membrane contact.

Different responses based on the interaction mechanisms were also observed in force spectroscopy. For example, the precipitous attraction upon the first contact between the membrane and the oil droplet was manifest for PVDF membrane but absent for the PDDA/SiNPs-PVDF membrane, which can be explained by their different surface wetting properties. The relatively significant electrostatic interaction between the positively charged PDDA/ SiNPs-PVDF membrane and the negatively charged oil droplet did not become effective until the oil droplet force probe was being retracted from the membrane surface.

The experimental evidences from CA measurements, force spectroscopy, and fouling experiments consistently lead to a cogent conclusion that surface potentials (charges) played a critical role, in addition to wettability, in determining the oil-membrane interaction in water. However, electrostatic interaction is a rather broad concept with many manifestations. For example, ionic bonding, electrical double layer interaction, or even the Keesom type of vdW interaction (e.g. hydrogen bond) can all be considered as electrostatic interaction [70]. The exact mechanism of electrostatic interaction relevant to our experimental observations in this study still needs to be explicated. In the following subsections, we will discuss the impact of the surface potentials on the oil-membrane interaction from both the DLVO and the interfacial tension perspectives.

\subsection{Mechanism of electrostatic interaction}

When particle-membrane interaction (colloidal deposition) is involved, the DLVO theory has been often cited for theoretical explanation [71-74]. The DLVO theory states that the affinity between a particle and a surface is primarily determined by the electrical double layer (EDL) interaction and the van der Waals (vdW) interaction. The attachment efficiency, defined as the probability for successful attachment per collision, has been employed to quantify such an affinity [74].

However, the applicability of the DLVO theory, for explaining the experimental results of membrane fouling in our study, is questionable. One major problem is that the EDL interaction, which comprises both osmotic and Coulombic contributions
$[70,75]$, should theoretically have negligible impact on the overall interaction due to the very short Debye length (calculated to be $\sim 0.4 \mathrm{~nm}$ for the feed solution used). Within such a short Debye length, the vdW force should dominate the overall interaction and eclipse any difference in the EDL interaction. Therefore, the EDL interaction was unlikely the manifestation of the differential electrostatic interactions between the PDA/SiNPs-PVDF and PDDA/ SiNPs-PVDF membranes that led to drastically different fouling behaviors.

To account for the impact of surface wetting properties, the extended DLVO ( $x$-DLVO) theory has often been cited to quantify the affinity between a particle and a membrane surface [76]. The $x$-DLVO theory incorporate the Lewis acid-base $(A B)$ interaction that accounts for the wetting property based short-range interactions such as hydration force. The short range vdW and $A B$ interactions contribute to the interfacial tension and the free energy of adhesion that not only determine how easy a particle can attach onto the membrane surface (i.e. the attachment efficiency) but also how readily a particle can detach from the membrane surface upon local perturbation.

According to van Oss, the $A B$ component of the interfacial tension involves interaction between electron acceptors and electron donors and only takes effect when the two interacting surfaces are both polar [76-78]. The surface charges of the membranes and the oil droplets have significant impact on the electron donor and electron acceptor parameters, which can explain the drastically different wetting, adhesion, and fouling behaviors between the positively charged PDDA/SiNPs-PVDF membrane and the negatively charged PDA/SiNPs-PVDF membrane. In other words, the electrostatic interaction most likely exerted its impact via the polar interaction component in surface adhesion, rather than the EDL interaction.

However, as free energy of adhesion is a thermodynamic property, it still cannot explain the dynamic behaviors of the PDA/ SiNPs-PVDF membrane in the CA measurement and force spectroscopy. We speculate that such dynamic wetting behavior involves the redistribution of natural anionic surfactants at the interface between crude oil and water. These anionic surfactants have lateral mobility along the interface and thus can migrate under the influence of electric field. When the oil droplet was in contact with a positively charged surface, these anionic surfactants gradually concentrated toward the contact area and thereby increased the local electronegativity, which resulted in a higher electron donor parameter of the oil surface and thus a stronger attractive $A B$ interaction between the oil droplet and the membrane.

\section{Conclusions}

In this study, we tested a hydrophobic PVDF membrane, a PDA/ SiNPs-PVDF composite membrane with a hydrophilic and negatively charged surface, and a PDDA/SiNPs-PVDF composite membrane with a hydrophilic and positively charged surface in membrane distillation experiments with a saline crude oil emulsion. While the fouling resistance of the PVDF membrane was expectably poor, both composite membranes were effective in fouling control, but to different extents. The negatively charged PDA/ SiNPs-PVDF membrane was highly effective and robust in mitigating the fouling of the negatively charged crude oil emulsion; whereas the positively charged PDDA/SiNPs-PVDF membrane only offered limited improvement over the hydrophobic PVDF membrane. The difference in the fouling behaviors between the three membranes was, to a high degree, corroborated by results obtained from underwater oil CA measurement and from oil droplet probe force spectroscopy. 
Dynamic behaviors of the wetting and adhesion were observed when the negatively charged crude oil droplet interacted with the hydrophilic but positively charge PDDA/SiNPs-PVDF membrane. The fundamental mechanism underlying this dynamic behavior remains uncertain. We also the relative irrelevance of the colloidal deposition approach and the strong relevance of the adhesion energy approach for elucidating the impact of electrostatic interaction on oil-membrane interaction. One possible explanation for the dynamic wetting and adhesion behaviors was the redistribution of charge baring surfactants at the oil water interface under the electrostatic influence of the oppositely charged membrane, which led to increasing attractive interaction between the crude oil droplet and the PDDA/SiNPs-PVDF membrane.

\section{Acknowledgements}

The authors acknowledge the financial support from Bureau of Reclamation, Department of Interior, via DWPR Agreement R15AC00088.

\section{Appendix A. Supplementary material}

Supplementary data associated with this article can be found in the online version at http://dx.doi.org/10.1016/j.memsci.2016.06. 011.

\section{References}

[1] M.K. Souhaimi, T. Matsuura, Membrane Distillation: Principles and Applications, Elsevier., Amsterdam, 2011.

[2] K.W. Lawson, D.R. Lloyd, Membrane distillation, J. Membr. Sci. 124 (1997) $1-25$.

[3] M. Khayet, T. Matsuura, Membr. Distill. (2011).

[4] S. Lin, N.Y. Yip, M. Elimelech, Direct contact membrane distillation with heat recovery: Thermodynamic insights from module scale modeling, J. Membr. Sci. 453 (2014) 498-515.

[5] E. Shaulsky, C. Boo, S. Lin, M. Elimelech, Membrane-based osmotic heat engine with organic solvent for enhanced power generation from low-grade heat, Environ. Sci. Technol. 49 (2015) 5820-5827.

[6] G. Zuo, R. Wang, R. Field, A.G. Fane, Energy efficiency evaluation and economic analyses of direct contact membrane distillation system using Aspen Plus, Desalination 283 (2011) 237-244.

[7] F. Banat, N. Jwaied, Economic evaluation of desalination by small-scale autonomous solar-powered membrane distillation units, Desalination 220 (2008) 566-573.

[8] S. Al-Obaidani, E. Curcio, F. Macedonio, G. Di Profio, H. Al-Hinai, E. Drioli, Potential of membrane distillation in seawater desalination: thermal efficiency, sensitivity study and cost estimation, J. Membr. Sci. 323 (2008) 85-98.

[9] J. Bundschuh, N. Ghaffour, H. Mahmoudi, M. Goosen, S. Mushtaq, J. Hoinkis, Low-cost low-enthalpy geothermal heat for freshwater production: innovative applications using thermal desalination processes, Renew. Sustain. Energy Rev. 43 (2015) 196-206.

[10] Y.D. Kim, K. Thu, N. Ghaffour, K. Choon Ng, Performance investigation of a solar-assisted direct contact membrane distillation system, J. Membr. Sci. 427 (2013) 345-364.

[11] N. Ghaffour, J. Bundschuh, H. Mahmoudi, M.F.A. Goosen, Renewable energydriven desalination technologies: a comprehensive review on challenges and potential applications of integrated systems, Desalination 356 (2014) 94-114.

[12] D.L. Shaffer, L.H. Arias Chavez, M. Ben-Sasson, S. Romero-Vargas Castrillón, N. Y. Yip, M. Elimelech, Desalination and reuse of high-salinity shale gas produced water: drivers, technologies, and future directions, Environ. Sci. Technol. 47 (2013) 9569-9583.

[13] S. Lin, S. Nejati, C. Boo, Y. Hu, C.O. Osuji, M. Elimelech, Omniphobic Membrane for Robust Membrane Distillation, Environ. Sci. Technol. Lett. 1 (2014) $443-447$.

[14] L.M. Camacho, L. Dumée, J. Zhang, J. De Li, M. Duke, J. Gomez, S. Gray, Advances in membrane distillation for water desalination and purification applications, Water 5 (2013) 94-196.

[15] J. Israelachvili, R. Pashley, The hydrophobic interaction is long range, decaying exponentially with distance, Nature 300 (1982) 341-342.

[16] Y. Tsao, D. Evans, H. Wennerstrom, Long-range attractive force between hydrophobic surfaces observed by atomic force microscopy, Science 262 (1993) 547-550.
[17] E.E. Meyer, K.J. Rosenberg, J. Israelachvili, Recent progress in understanding hydrophobic interactions, Proc. Natl. Acad. Sci. U. S. A. 103 (2006) 15739-15746.

[18] Z. Wang, D. Hou, S. Lin, Composite membrane with underwater-oleophobic surface for anti-oil-fouling membrane distillation, Environ. Sci. Technol. 50 (2016) 3866-3874.

[19] G. Zuo, R. Wang, Novel membrane surface modification to enhance anti-oil fouling property for membrane distillation application, J. Membr. Sci. 447 (2013) 26-35.

[20] S. Kim, D.W. Lee, J. Cho, Application of direct contact membrane distillation process to treat anaerobic digestate, J. Membr. Sci. 511 (2016) 20-28.

[21] J. Genzer, K. Efimenko, Recent developments in superhydrophobic surfaces and their relevance to marine fouling: a review, Biofouling 22 (2006) 339-360.

[22] S. Pechook, K. Sudakov, I. Polishchuk, I. Ostrova, V. Zakin, B. Pokroy, M. Shemesh, Bioinspired passive anti-biofouling surfaces preventing biofilm formation, J. Mater. Chem. B 4 (2014) 1166-1169.

[23] S. Nishimoto, B. Bhushan, Bioinspired self-cleaning surfaces with superhydrophobicity, superoleophobicity, and superhydrophilicity, RSC Adv. (2012) 671-690.

[24] M. Liu, S. Wang, Z. Wei, Y. Song, L. Jiang, Bioinspired design of a superoleophobic and low adhesive water/solid interface, Adv. Mater. 21 (2009) 665-669.

[25] X. Liu, J. Zhou, Z. Xue, J. Gao, J. Meng, S. Wang, L. Jiang, Clam's shell inspired high-energy inorganic coatings with underwater low adhesive superoleophobicity, Adv. Mater. 24 (2012) 3401-3405.

[26] Z. Xue, S. Wang, L. Lin, L. Chen, M. Liu, L. Feng, L. Jiang, A novel superhydrophilic and underwater superoleophobic hydrogel-coated mesh for oil/ water separation, Adv. Mater. 23 (2011) 4270-4273.

[27] Q. Wen, J.C. Di, L. Jiang, J.H. Yu, R.R. Xu, Zeolite-coated mesh film for efficient oil-water separation, Chem. Sci. 4 (2013) 591-595.

[28] Y. Zhu, F. Zhang, D. Wang, X.F. Pei, W. Zhang, J. Jin, A novel zwitterionic polyelectrolyte grafted PVDF membrane for thoroughly separating oil from water with ultrahigh efficiency, J. Mater. Chem. A 1 (2013) 5758-5765.

[29] K. Rohrbach, Y. Li, H. Zhu, Z. Liu, J. Dai, J. Andreasen, L. Hu, A cellulose based hydrophilic, oleophobic hydrated filter for water/oil separation, Chem. Commun. 50 (2014) 13296-13299.

[30] V. Hejazi, A.E. Nyong, P.K. Rohatgi, M. Nosonovsky, Wetting transitions in underwater oleophobic surface of brass, Adv. Mater. 24 (2012) 5963-5966.

[31] S.S. Chhatre, A. Tuteja, W. Choi, A. Revaux, D. Smith, J.M. Mabry, G.H. McKinley, R.E. Cohen, Thermal annealing treatment to achieve switchable and reversible oleophobicity on fabrics, Langmuir 25 (2009) 13625-13632.

[32] P.S. Brown, O.D.L. a Atkinson, J.P.S. Badyal, Ultrafast oleophobic-hydrophilic switching surfaces for antifogging, self-cleaning, and oil-water separation, ACS Appl. Mater. Interfaces 6 (2014) 7504-7511.

[33] Z. Wang, M. Elimelech, S. Lin, Environmental applications of interfacial materials with special wettability, Environ. Sci. Technol. 50 (2016) 2132-3150.

[34] R.M. Pashley, Hydration forces between mica surfaces in aqueous electrolyte solutions, J. Colloid Interface Sci. 80 (1981) 153-162.

[35] R.M. Pashley, DLVO and hydration forces between mica surfaces in $\mathrm{Li}+, \mathrm{Na}+$, $\mathrm{K}+$, and Cs + electrolyte solutions: a correlation of double-layer and hydration forces with surface cation exchange properties, J. Colloid Interface Sci. 83 (1981) 531-546.

[36] M. Rinaudo, Chitin and chitosan: properties and applications, Prog. Polym. Sci. 31 (2006) 603-632.

[37] D. Anjali Devi, B. Smitha, S. Sridhar, T.M. Aminabhavi, Pervaporation separation of isopropanol/water mixtures through crosslinked chitosan membranes, J. Membr. Sci. 262 (2005) 91-99.

[38] S. Chen, L. Li, C. Zhao, J. Zheng, Surface hydration: principles and applications toward low-fouling/nonfouling biomaterials, Polym. (Guildf. ) 51 (2010) 5283-5293.

[39] A.V. Dudchenko, J. Rolf, L. Shi, L. Olivas, W. Duan, D. Jassby, Coupling underwater superoleophobic membranes with magnetic pickering emulsions for fouling-free separation of crude oil/water mixtures: an experimental and theoretical study, ACS Nano. 9 (2015) 9930-9941.

[40] H. Fe, D. Lu, W. Cheng, T. Zhang, X. Lu, Q. Liu, J. Jiang, P. Technology, W. Cheng, T. Zhang, X. Lu, Q. Liu, J. Jiang, H. Fe, Hydrophilic $\mathrm{Fe}_{2} \mathrm{O}_{3}$ dynamic membrane mitigating fouling of support ceramic membrane in ultrafiltration of oil/water emulsion, Sep. Purif. Technol. 165 (2016) 1-9.

[41] P.-C. Chen, Z.-K. Xu, Mineral-coated polymer membranes with superhydrophilicity and underwater superoleophobicity for effective oil/water separation, Sci. Rep. 3 (2013) 2776.

[42] H. Shi, Y. He, Y. Pan, H. Di, G. Zeng, L. Zhang, C. Zhang, A modified musselinspired method to fabricate $\mathrm{TiO}_{2}$ decorated superhydrophilic PVDF membrane for oil/water separation, J. Membr. Sci. 506 (2016) 60-70.

[43] J. Zeng, Z. Guo, Superhydrophilic and underwater superoleophobic MFI zeolite-coated film for oil/water separation, Colloids, Surf. A Physicochem. Eng. ASP 444 (2014) 283-288.

[44] C.J. Van Oss, M.K. Chaudhury, R.J. Good, Interfacial Lifshitz-van der Waals and polar interactions in macroscopic systems, Chem. Rev. 88 (1988) 927-941.

[45] C.J. van Oss, R.F. Giese, P.M. Costanzo, DLVO and Non-DLVO Interactions in Hectorite, Clays Clay Miner. 38 (1990) 151-159.

[46] C.J. van Oss, Acid-base interfacial interactions in aqueous media, Colloids, Surf. A Physicochem. Eng. ASP 78 (1993) 1-49.

[47] H.-C. Yang, K.-J. Liao, H. Huang, Q.-Y. Wu, L.-S. Wan, Z.-K. Xu, Mussel-inspired modification of a polymer membrane for ultra-high water permeability and oil-in-water emulsion separation, J. Mater. Chem. A 2 (2014) 10225. 
[48] N. Liu, Mussel power, Nature 7 (2008) 2-3.

[49] P.S. Brown, B. Bhushan, Mechanically durable, superomniphobic coatings prepared by layer-by-layer technique for self-cleaning and anti-smudge, J. Colloid Interface Sci. 456 (2015) 210-218.

[50] M. Khayet, T. Matsuura, Preparation and characterization of polyvinylidene fluoride membranes for membrane distillation, Ind. Eng. Chem. 40 (2001) 5710-5718.

[51] K. Smolder, A. Franken, Terminology for membrane distillation, Desalination 72 (1989) 249-262.

[52] D. Hou, G. Dai, J. Wang, H. Fan, L. Zhang, Z. Luan, Preparation and characterization of PVDF/nonwoven fabric flat-sheet composite membranes for desalination through direct contact membrane distillation, Sep. Purif. Technol. 101 (2012) 1-10.

[53] D. Hou, G. Dai, J. Wang, H. Fan, Z. Luan, C. Fu, Boron removal and desalination from seawater by PVDF flat-sheet membrane through direct contact membrane distillation, Desalination 326 (2013) 115-124.

[54] H. Xie, T. Saito, M.A. Hickner, Zeta potential of ion-conductive membranes by streaming current measurements, Langmuir 27 (2011) 4721-4727.

[55] A.V. Delgado, F. González-Caballero, R.J. Hunter, L.K. Koopal, J. Lyklema, Measurement and interpretation of electrokinetic phenomena, J. Colloid Interface Sci. 309 (2007) 194-224.

[56] K. Liu, J. Du, J. Wu, L. Jiang, Superhydrophobic gecko feet with high adhesive forces towards water and their bio-inspired materials, Nanoscale 4 (2012) 768 .

[57] X. Yao, J. Gao, Y. Song, L. Jiang, Superoleophobic surfaces with controllable oil adhesion and their application in oil transportation, Adv. Funct. Mater. 21 (2011) 4270-4276.

[58] L. Heng, J. Su, J. Zhai, Q. Yang, L. Jiang, Dual high adhesion surface for water in air and for oil underwater, Langmuir 27 (2011) 12466-12471.

[59] R.N. Wenzel, Resistance of solid surfaces to wetting by water, J. Ind. Eng. Chem. (Wash. D. C.) 28 (1936) 988-994.

[60] R.N. Wenzel, Surface roughness and contact angle, J. Phys. Colloid Chem. 53 (1949) 1466-1467.

[61] M. Elimelech, W.H. Chen, J.J. Waypa, Measuring the zeta (electrokinetic) potential of reverse osmosis membranes by a streaming potential analyzer, Desalination 95 (1994) 269-286.

[62] K. Boussu, Y. Zhang, J. Cocquyt, P. Van der Meeren, A. Volodin, C. Van Haesendonck, J.A. Martens, B. Van der Bruggen, Characterization of polymeric nanofiltration membranes for systematic analysis of membrane performance, J. Membr. Sci. 278 (2006) 418-427.

[63] J. a Howarter, J.P. Youngblood, Amphiphile grafted membranes for the separation of oil-in-water dispersions, J. Colloid Interface Sci. 329 (2009) 127-132.

[64] J. a Howarter, K.L. Genson, J.P. Youngblood, Wetting behavior of oleophobic polymer coatings synthesized from fluorosurfactant-macromers, ACS Appl. Mater. Interfaces 3 (2011) 2022-2030.

[65] D. Langevin, S. Poteau, I. Hénaut, J.F. Argillier, Crude oil emulsion properties and their application to heavy oil transportation, Oil Gas. Sci. Technol. 59 (2004) 511-521.

66] A.P. Sullivan, P.K. Kilpatrick, The effects of inorganic solid particles on water and crude oil emulsion stability, Ind. Eng. Chem. Res. 41 (2002) 3389-3404.

[67] L. Martínez-Díez, M. Vázquez-González, Temperature and concentration polarization in membrane distillation of aqueous salt solutions, J. Membr. Sci. 156 (1999) 265-273.

[68] M. Qtaishat, T. Matsuura, B. Kruczek, M. Khayet, Heat and mass transfer analysis in direct contact membrane distillation, Desalination 219 (2008) 272-292.

[69] D.L. Shaffer, J.R. Werber, H. Jaramillo, S. Lin, M. Elimelech, Forward osmosis: where are we now? Desalination 356 (2014) 271-284.

[70] J. Israelachvili, Intermolecular and Surface Forces, 3rd Edition, 2010.

71] J.A. Brant, A.E. Childress, Colloidal adhesion to hydrophilic membrane surfaces, J. Membr. Sci. 241 (2004) 235-248.

[72] E.M.V. Hoek, S. Bhattacharjee, M. Elimelech, Effect of membrane surface roughness on colloid-membrane DLVO interactions, Langmuir 19 (2003) 4836-4847.

[73] A. Subramani, E.M.V. Hoek, Direct observation of initial microbial deposition onto reverse osmosis and nanofiltration membranes, J. Membr. Sci. 319 (2008 111-125.

[74] M. Elimelech, X. Jia, J. Gregory, R. Williams, Particle Deposition and Aggregation, Butterworth-Heinemann,, 1998.

[75] S. Lin, M.R. Wiesner, Paradox of stability of nanoparticles at very low ionic strength, Langmuir 28 (2012) 11032-11041.

[76] J.A. Brant, A.E. Childress, Assessing short-range membrane-colloid interactions using surface energetics, J. Membr. Sci. 203 (2002) 257-273.

[77] A. Tiraferri, Y. Kang, E.P. Giannelis, M. Elimelech, Superhydrophilic thin-film composite forward osmosis membranes for organic fouling control: Fouling behavior and antifouling mechanisms, Environ. Sci. Technol. 46 (2012) 11135-11144.

[78] C. Van Oss, R. Good, M. Chaudhury, C. Oss, Additive and nonadditive surface tension components and the interpretation of contact angles, Langmuir 4 (1988) 884-891. 